\title{
Estudo retrospectivo (histórico) da dengue no Brasil: características regionais e dinâmicas
}

\section{Regional and dynamics characteristics of dengue in Brazil: a retrospective study}

\author{
Fernando Portela Câmara ${ }^{1}$, Regina Lúcia Gonçalves Theophilo ${ }^{1}$, Gualberto Teixeira dos \\ Santos $^{2}$, Silvia Regina Ferreira Gonçalves Pereira ${ }^{3}$, Daniel Cardoso P. Câmara ${ }^{1}$ \\ e Roberto Rodrigues C. de Matos ${ }^{1}$
}

\begin{abstract}
RESUMO
A dengue no Brasil incide tipicamente nos meses mais quentes do ano, sem diferenças qualitativas para as regiões brasileiras, porém, com diferenças quantitativas importantes, dividindo o país em dois grupos distintos quanto ao número de notificações de casos. Oprimeiro grupo compreende as regiões Nordeste e Sudeste, que deteve cerca de $86 \%$ das notificações, enquanto o segundo (regiões Sul, Centro-Oeste e Norte) é responsável por um número significativamente menor. Os índices vetoriais estavam associados primariamente ao tamanho das populações, sendo mais freqüentes os índices abaixo do valor de risco e ainda assim, nesta condição, ocorreram epidemias. Não foi observada correlação positiva entre epidemias e densidades vetoriais.
\end{abstract}

Palavras-chaves: Dengue. Epidemiologia da dengue. Dengue nas regiões do Brasil. Modelos preditivos em dengue.

\section{ABSTRACT}

Dengue epidemics occur typically in the warmest months without qualitative differences for the Brazilian geographic regions. However, this disease has an important quantitative difference. We observe two clusters in the country regarding the number of case notifications: The first cluster is formed by the North-East and South-East regions, that sum about 86\% of the all notifications; and the second is formed by the South, North and Center-West regions. Vector index were associated to the population sizes, and most of them were below of the recognized threshold index for epidemic's risk although epidemic situation were observable in some of these places. Apparently, there was no positive evidence between dengue's epidemics and vector index.

Key-words: Dengue. Dengue epidemiology. Dengue at the Brazilian regions. Predictive models in dengue.

A dengue é hoje objeto da maior campanha de saúde pública do Brasil, que se concentra no controle do Ae aegypti, único vetor reconhecido como transmissor do vírus da dengue em nosso meio. Este mosquito está adaptado a se reproduzir nos ambientes doméstico e peridoméstico ${ }^{21}$, utilizando-se de recipientes que armazenam água potável e recipientes descartáveis que acumulam água de chuvas, comumente encontrados nos lixos das cidades ${ }^{21}$. A dengue encontra-se hoje presente em todos os 27 estados da Federação $0^{18}$, distribuída por 3.794 municípios $^{15}$, sendo responsável por cerca de $60 \%$ das notificações nas Américas.

A introdução seqüencial de diferentes sorotipos do vírus da dengue contribui para pressionar a incidência desta doença. Em 1981, os sorotipos DEN-1 e DEN 4 foram os primeiros a serem isolados em uma epidemia de dengue ocorrida Boa Vista, Estado de Roraima ${ }^{14}$. Após um silêncio epidemiológico, o sorotipo DEN-1 invadiu o Sudeste (Rio de Janeiro) e Nordeste (Alagoas, Ceará, Pernambuco, Bahia, Minas Gerais) em 1986-1987 $7^{217}$, espalhando pelo país desde então, com as entradas dos sorotipos DEN-2 em 1990-1991 ${ }^{13}$, e o DEN-3 em 2001-2002 ${ }^{12}$. No momento, estes três sorotipos circulam simultaneamente em 24 estados da Federação ${ }^{18}$, contribuindo para a incidência das formas graves da dengue (dengue hemorrágica e síndrome do choque da dengue) nas cidades onde se registraram epidemias sequenciais por pelo menos dois sorotipos diferentes 5 , embora a virulência da cepa epidêmica possa ser algumas vezes o determinante principal das formas hemorrágicas ${ }^{101620}$.

A progressão da dengue depende de condições ecológicas e sócio-ambientais que facilitam a dispersão do vetor. Na ausência de uma vacina eficaz, o controle da transmissão do vírus da

\footnotetext{
1. Setor de Epidemiologia de Doenças Infecciosas, Departamento de Virologia, Instituto de Microbiologia Prof. Paulo de Góes, Universidade Federal do Rio de Janeiro, Rio de Janeiro, RJ. 2. Assessoria de Doenças Transmitidas por Vetores e Zoonoses, Secretaria Estadual de Saúde do Rio de Janeiro, Rio de Janeiro, RJ. 3. Laboratório de Sanidade Animal, Centro de Ciências e Tecnologias Agropecuárias, Universidade Estadual do Norte Fluminense Darcy Ribeiro, Campos, RJ. Projeto financiado pelo MS/SUS/CNPq/UNESCO, processo \# 501553/2003-7 e SUS/FAPERJ processo \# E-26/170.621/2005 Endereço para correspondência: Dr. Fernando Portela Câmara. Rua Pinheiro Machado 25/405, Laranjeiras, 22231-090 Rio de Janeiro, RJ e-mail:portela@micro.ufrj.br Recebido para publicação em 9/5/2006 Aceito em 2/4/2006
} 
dengue requer o esforço conjunto de toda a sociedade no combate ao vetor. Dada a extraordinária capacidade de adaptação do Ae aegypti ao ambiente, esta tarefa nem sempre produz resultados previsíveis.

Neste trabalho, mostramos o comportamento histórico da dengue segundo as regiões brasileiras, e as relações entre casos notificados da doença, tamanho de populações e densidade vetorial. Trata-se, portanto, de um estudo ecológico longitudonal. Com isso, procuramos definir parâmetros a partir da experiência nacional que possam contribuir racionalmente para o combate ao vetor, que também é o mais premente fator de risco para a reurbanização da febre amarela.

\section{MATERIAL E MÉTODOS}

Área de estudo. Abrangeu as cinco regiões geográficas (Sul, Sudeste, Centro-Oeste, Nordeste e Norte) do território brasileiro $^{6}$. A região Sul (6,8\% do território nacional) compreende os Estados do Paraná (PR), Santa Catarina (SC) e Rio Grande do Sul (RS), com população superior a 26 milhões de habitantes, $80,9 \%$ vivendo no meio urbano, e densidade demográfica de 43,5 habitantes/ $\mathrm{km}^{2}$. 0 clima é subtropical, exceto no norte do Paraná, de clima tropical. A região Sudeste $(10,9 \%$ do território nacional) compreende os estados de Minas Gerais (MG), Espírito Santo (ES), Rio de Janeiro (RJ) e São Paulo (SP), com população de cerca de 77 milhões de habitantes, com maior densidade demográfica $\left(78,1\right.$ habitantes $\left./ \mathrm{km}^{2}\right)$ e maior índice de urbanização $(90,5 \%)$ do país. Região montanhosa com clima tropical atlântico no litoral e tropical de altitude nos planaltos. Abriga as duas mais importantes metrópoles nacionais, as Cidades de São Paulo e Rio de Janeiro, tendo ainda Belo Horizonte (MG) como importante metrópole regional. A economia desta região é a mais desenvolvida e industrializada do país, concentrando mais da metade da produção nacional. É também o principal pólo turístico e comercial do país. A região Centro-Oeste (18,9\% do território nacional) compreende os Estados de Goiás (G0), Mato Grosso (MT), Mato Grosso do Sul (MS) e Distrito Federal (DF), população aproximada de 12 milhões de habitantes, 81,3\% concentrando-se na zona urbana, com densidade demográfica de 6,5 habitantes $/ \mathrm{km}^{2}$. Localiza-se no Planalto Central e tem clima tropical semi-úmido, com freqüentes chuvas de verão.

A região Nordeste (18,2\% do território nacional), tem cerca de 50 milhões de habitantes e compreende os Estados do Maranhão (MA), Piauí (PI), Ceará (CE), Rio Grande do Norte (RN), Paraíba (PB), Pernambuco (PE), Alagoas (AL), Sergipe (SE) e Bahia (BA). Subdivide-se em zona da mata, zona do agreste, sertão e polígono das secas. A zona da mata é uma faixa litorânea de $200 \mathrm{~km}$, com clima tropical úmido e chuvas predominando no outono e inverno, sendo a zona mais povoada, urbanizada e industrializada da região. É zona de praias, com turismo em ascensão. A zona do agreste é zona de transição, húmida. 0 sertão é de clima semi-árido, com chuvas escassas, expandindo-se do interior até o litoral do RN e CE. 0 polígono das secas é a área de estiagem que poupa apenas o MA e a faixa litorânea.
A região Norte (45,2\% do território nacional), tem clima equatorial e compreende os Estados do Acre (AC), Amazonas (AM), Roraima (RR), Rondônia (R0), Pará (PA), Amapá (AP) e Tocantins (TO), compondo uma região formada pelas grandes bacias dos rios Amazonas e Tocantins e pela floresta tropical Amazônica. Tem cerca de 14 milhões de habitantes, 57,8\% na área urbana, e a densidade demográfica mais baixa do país $(2,9$ habitantes $/ \mathrm{km}^{2}$ ). Belém (PA) é a mais importante metrópole desta região.

Fonte dos dados e análise estatística. As séries históricas de cada região brasileira foram obtidas da Fundação Nacional de Saúde (FUNASA), compreendendo o período de 1986 a 2003. As séries históricas para o Rio de Janeiro foram obtidas da Assessoria de Doenças Transmitidas por Vetores e Zoonoses (SES-RJ/SS/CVE/Assessoria de Doenças Transmitidas por Vetores e Zoonoses). Os dados epidemiológicos do triênio 2001-2003, para o Rio de Janeiro, foram obtidos do Sistema de Informações de Agravos de Notificação, Secretaria de Estado de Saúde do Estado do Rio de Janeiro (SINAM) e os respectivos índices vetoriais do Sistema de Informação de Febre Amarela e Dengue, Secretaria de Estado de Saúde do estado do Rio de Janeiro (SISFAD). Utilizou-se o software Minitab 14.0 para gerar informação gráfica e para as análises estatísticas citadas no texto.

\section{RESULTADOS}

Características regionais da dengue. Para caracterizar os períodos dos picos e remissões das epidemias no período anual, utilizamos as médias trimestrais acumuladas das notificações por região (Figura 1). A maioria das notificações concentrouse no primeiro semestre do ano, correspondente aos meses mais quentes. Nas regiões Sudeste, Centro-Oeste e Sul, mais da metade dos casos ocorreram no primeiro trimestre. Na região Nordeste, a maior concentração das notificações incidiram no segundo trimestre. As notificações para cada região eram sempre significativamente maiores na primeira metade do ano, quando comparadas à segunda (testes t para cada região, $0,004 \leq \mathrm{p} \leq 0,027)$.

A região Nordeste deteve o maior (48,3\%) número de casos notificados do total, seguido pelas regiões Sudeste $(37,2 \%)$, Centro-Oeste $(7,6 \%)$, Norte $(5,7 \%)$ e Sul $(1,2 \%)$. Juntas, as regiões Nordeste e Sudeste concorreram para $85,5 \%$ dos casos notificados de dengue. A análise de variância mostrou uma diferença significativa no conjunto das cinco regiões $(\mathrm{p}<0,001)$, separando dois grupos distintos: o primeiro, constituído pelas regiões Sudeste e Nordeste, concentrando os maiores níveis de notificações, e o segundo, constituído pelas três outras regiões, concentrando níveis significativamente menores.

Dinâmica da dengue em populações expostas ao vetor. Para analisar a relação entre tamanho de populações e notificações de dengue, utilizamos os dados entomológicos dos 92 municípios do Estado do Rio de Janeiro, referentes período epidêmico de 2001-2003, devido à confiabilidade dos mesmos. 


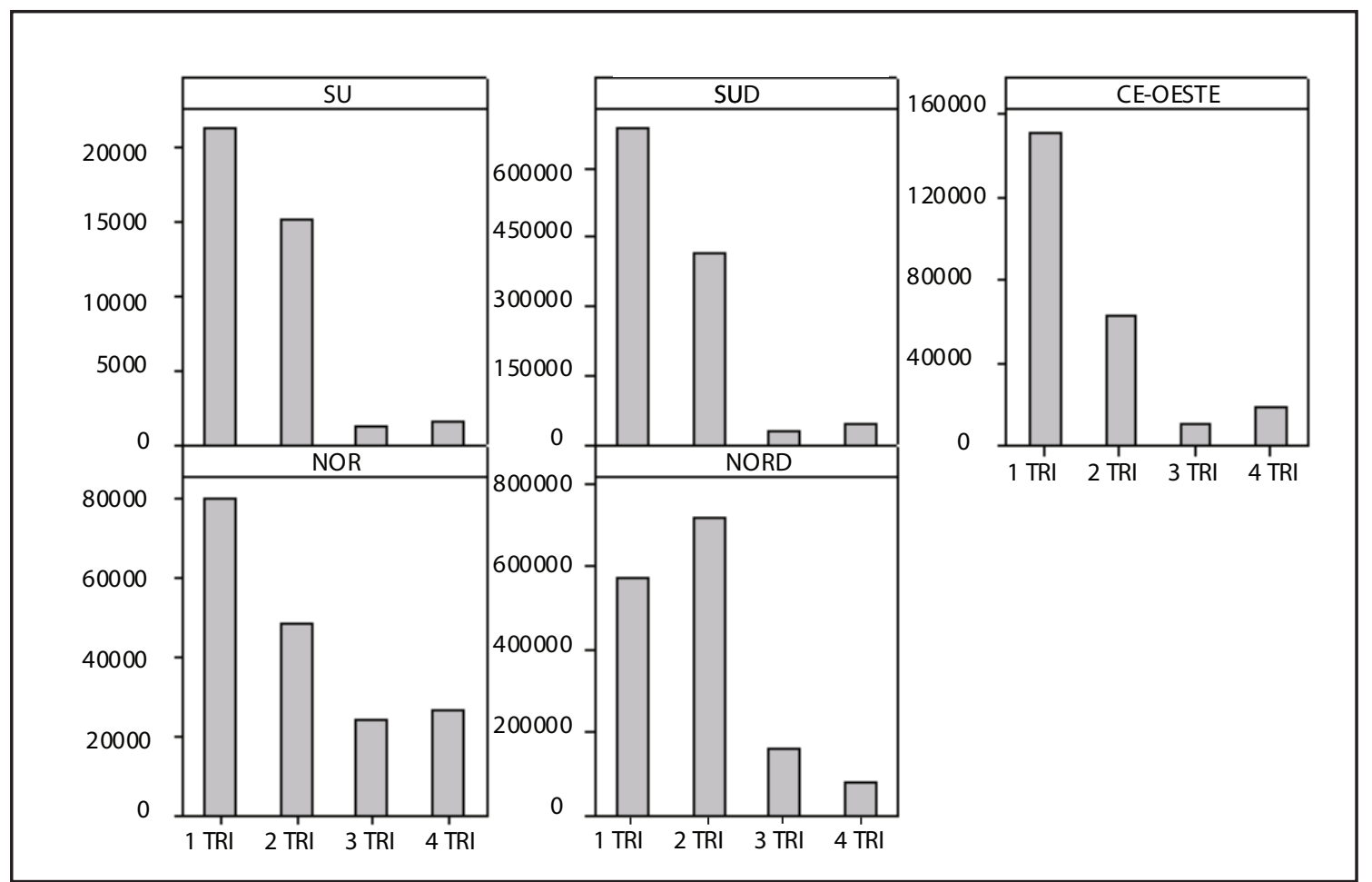

Figura 1 - Característica da dengue nas regiões brasileiras segundo as notificações acumuladas por trimestre. Note que a doença incide nos meses quentes, com picos no primeiro trimestre do ano, exceto para a região Nordeste, com maior notificação de casos no segundo trimestre.

Os dados (população do município e casos notificados nos anos mencionados) foram transformados em valores logarítmicos, obtendose distribuições normais, e então analisados parametricamente. As correlações foram altamente significativas para $2001(\mathrm{r}=0,78$, $\mathrm{p}<0,001), 2002(\mathrm{r}=0,86, \mathrm{p}<0,001)$ e 2003 ( $\mathrm{r}=0,71$, $\mathrm{p}<0,001$ ). A análise dos resíduos de cada regressão revelou um típico padrão randômico, indicando que os modelos capturavam toda a estrutura dos dados. A Figura 2 mostra o modelo de regressão linear (Log de Casos = - 3,57 + 1,31 Log da População) para o ano de 2002, período em que a epidemia teve seu maior número de casos. 0 modelo explicou $73 \%$ da variabilidade dos casos.
Relação entre índices vetoriais e incidência de dengue. Utilizamos também os dados acima referidos para modelo geral. Não encontramos diferenças estatisticamente significativas entre os índices no triênio considerado (ANOVA), e os índices vetoriais correlacionavam-se significativamente entre si $(0,56 \leq \mathrm{r} \leq 0,83, \mathrm{p}<0,001)$. A distribuição de índices vetoriais por número de municípios do estado nos anos de 2001-2003 (Figura 3), revelou que a maioria dos municípios tinha índices vetoriais na faixa de 0 a 1 , embora estivessem em situação epidêmica. Esta faixa de índice foi mantida nos mesmos municípios durante o triênio.
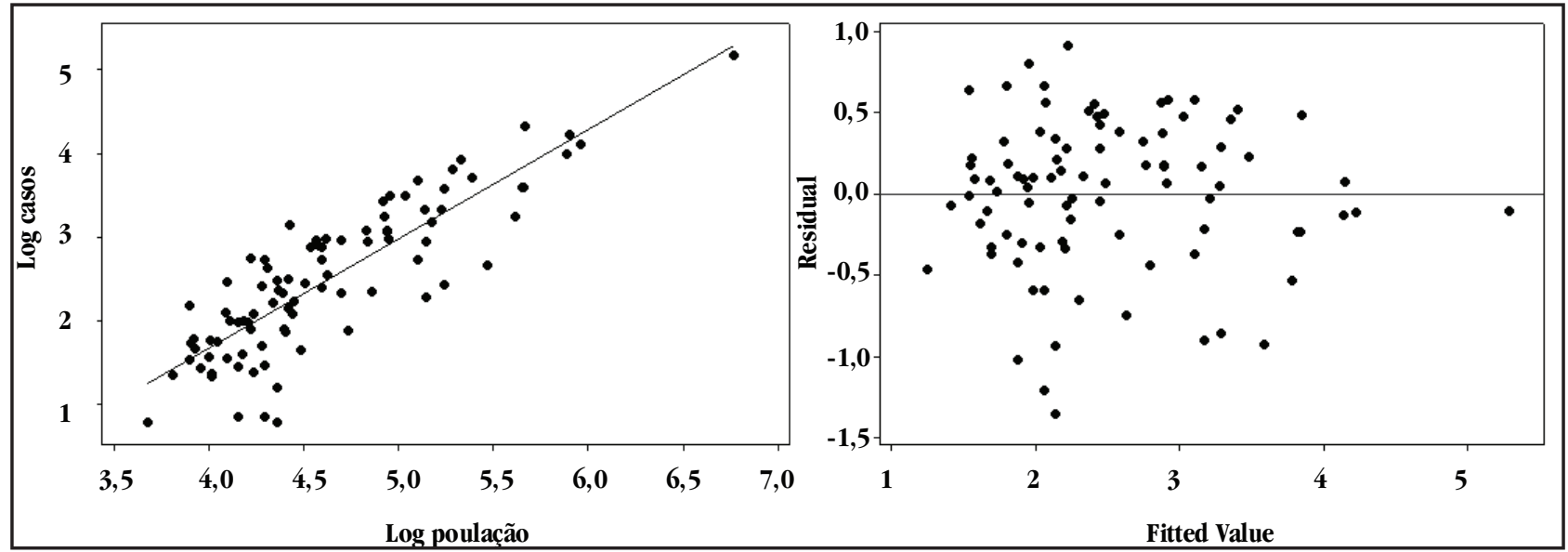

Figura 2 - Análise de regressão (linear, bivariada) relacionando tamanhos de populações expostas (log) e casos de dengue (log) para 2002 , ano de maior atividade epidêmica para o Estado do Rio de Janeiro. 0 modelo, Log de Casos = - 3,57 + 1,31 Log da População, capturou toda estrutura dos dados e explicou $73 \%$ da variância da variável resposta. 


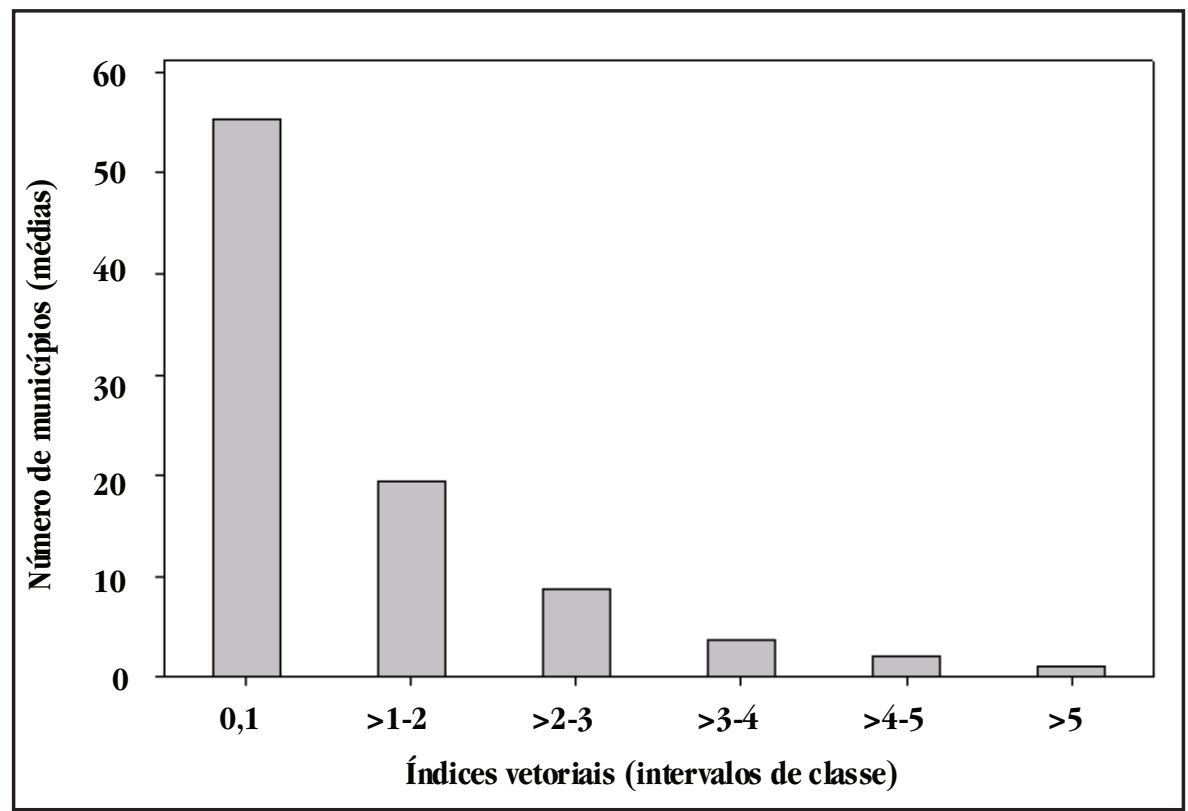

Figura 3 - Distribuição das médias dos índices vetoriais nos municípios do Estado do Rio de Janeiro (2001-2003). Note que a maioria apresentava indices de infestação predial entre 0 e 1.

\section{DISCUSSÃO}

No período da última grande epidemia de 2001-2003, foram notificados 1.564 .112 casos de dengue no país, sendo 4.123 na forma hemorrágica, com 217 óbitos $^{19}$. Se considerarmos que estas notificações representam apenas cerca de $15 \%$ do total notificado ${ }^{8}$ é possível que o numero de casos tenha sido da ordem de 10 milhões. Além disso, se considerarmos ainda que grande parte das infecções pelo vírus da dengue é assintomática ${ }^{22}$, o número real de casos pode ter sido superior a 40 milhões, cerca de $20 \%$ da população do país. É, pois, de considerável relevância combater esta doença em nosso meio.

A significativa incidência sazonal da dengue nos meses quentes (Figura 1) associa-se à conhecida sensibilidade do ciclo reprodutivo do Ae. aegypti a variações de temperaturas ${ }^{1}$. Nos meses em que a temperatura cai, na segunda metade do ano, verifica-se que a incidência de casos diminui significativamente, como é bem conhecido. Contudo, isto não concorre para interromper a transmissão.

Embora a dengue tenha se revelado qualitativamente semelhante em todas as regiões do país, quantitativamente apresentou diferenças importantes. Neste último caso, foi possível separar dois grupos distintos (ANOVA): o primeiro, formado pelas regiões Nordeste e Sudeste, deteve $86 \%$ de todas as notificações, e o segundo, formado pelas regiões Norte, Sul e Centro-Oeste, uma pequena proporção restante. No primeiro grupo, estão os principais pólos atratores ou cidades que favorecem a disseminação do vírus e do vetor para o país. Estas cidades atraem trabalhadores, turistas e visitantes que aí se expõe à infecção e levam o vírus para seus locais de origem ao retornarem. Também, as numerosas rotas de tráfego que irradiam destes pólos concorrem para a rápida disseminação do vírus e do vetor. É possível que uma intensa e constante campanha nestes atratores possa repercutir em todas as demais partes do país, pois, a interrupção da circulação de vírus para fora destes polos poderá fazer retroceder gradualmente a incidência em muitos dos lugares em que a manutenção do vírus não se sustenta por si mesma. De fato, a manutenção do vírus da dengue pode não depender apenas dos grandes criadouros de $A \boldsymbol{e}$. aegypti, mas de uma tamanho crítico da população que garanta a sustentação do vírus na mesma ${ }^{2}$.

0 número de casos estava associado ao tamanho da população, e não aos índices vetoriais. De fato, a maioria (56\%) dos municípios que apresentou índice vetorial abaixo do valor preconizado para risco de epidemia (Figura 3), estava em situação epidêmica. Não houve como relacionar este fato como casos de dengue não autóctones, isto é, infecções adquiridas em cidades pólos e então trazidas para as cidades onde os infectados são moradores e menos expostos ao vetor. Entretanto, este fato corrobora com resultados descritos por outros autores que negam haver correlação significativa entre densidade de Ae. aegypti e epidemias de dengue ${ }^{3}$, bastando apenas a presença do vetor na comunidade. Morrison e cols ${ }^{11}$ não consideram importante a magnitude de densidade do vetor nas epidemias, bastando apenas que haja 10 a 20 de fêmeas de Ae aegypti por residência, e que uma ou duas delas sejam vetores.

A complexidade dos fatos aqui apresentados sugerem que a vacina antidengue, ainda não existente, talvez seja a opção mais segura para o controle desta arbovirose urbana.

\section{AGRADECIMENTOS}

Ao técnico Fernando Gomes da Costa (com bolsa de apoio técnico do $\mathrm{CNPq}$ ) pelo auxílio prestado. 


\section{REFERÊNCIAS}

1. Consoli RAGB, Oliveira RL. Principais Mosquitos de Importância Sanitária no Brasil Rio de Janeiro, Editora da Fundação Oswaldo Cruz, Rio de Janeiro, 1994

2. Donalísio MR. O dengue no espaço habitado. Editora Humanismo, Ciência e Tecnologia, São Paulo, 1999.

3. Focks DA, Daniels E, Haile DG, Keesling JE. A simulation model of the epidemiology of urban dengue fever: literature analysis, model development, preliminary validation, and samples of simulation results. American Journal of Tropical Medicine and Hygiene 53: 489-506, 1995.

4. Fundação Nacional de Saúde. http://sis.funasa.gov.br/, acessado em 24.10.2005, 2005.

5. Halstead SB. Global epidemiology of Dengue Hemorrhagic Fever. Southeast Asian Journal of Tropical Medicine and Public Health 21: 636-641, 1990.

6. Instituto Brasileiro de Geografia e Estatística. http://www.ibge.gov.br/, acesssado em 24.10.2005, 2005.

7. Kermack WD, McKendrick AG. A contribution to the mathematical theory of epidemics. Journal of Royal Statistical Society 115: 700-721, 1929.

8. Lima VLC, Figueiredo LTM, Correa F HR, Leite OF, Rangel O, Vido AA, Oliveira SS, Owa MA, Carlucci RH. Dengue: inquérito sorológico pós epidemiológico em zona urbana do estado de São Paulo, Brasil. Revista Saúde Pública 33: 566-574, 1999.

9. Ministério da Saúde. Guia de Vigilância Epidemiológica, 6 a edição, Secretaria de Vigilância Sanitária. Brasília, 2005.

10. Morens DM, Marchette NJ, Chu MC, Halstead SB. Growth of dengue type 2 virus isolates in human peripheral blood leukocytes correlates with severe and mild dengue disease. American Journal of Tropical Medicine and Hygiene 45: 644-651, 1991.

11. Morrison AC, Getis A, Santiago M. Exploratory space-time analysis of reported dengue cases during an outbreak in Florida, Puerto Rico, 1991-1992. American Journal of Tropical Medicine and Hygiene 57: 119-125, 1997.
12. Nogueira RMR, Miagostovich MP, Filipis AMP, Pereira MAS, Schatzmayr HG. Dengue virus type 2 in Rio de Janeiro, Brazil. Memórias do Instituto Oswaldo Cruz 96: 925-926, 2001

13. Nogueira RMR, Zagner SMO, Martins ISM, Lampe E, Miagostovich MP, Schatzmayr HG. Dengue Heamorrhagic Fever/Dengue Shock Syndrome (DHF/DSS) caused by serotype 2 in Brazil. Memórias do Instituto Oswaldo Cruz 86: 269, 1991.

14. Osanai CH. A epidemia de dengue em Boa Vista, Território Federal de Roraima, 1981-1982. Dissertação de mestrado, Escola Nacional de Saúde Pública, Rio de Janeiro, RJ, 1984

15. Pimenta Jr FG. Instrumento para avaliar a implantação do Programa Naciona de Controle do Dengue no âmbito municipal, Dissertação de Mestrado Escola Nacional de Saúde Pública, Rio de Janeiro, RJ, 2005.

16. Rosen L. disease exacerbation caused by sequential dengue infections: Myth or reality? Review Infectious Diseases 11 (supl 4): S840-S842, 1989

17. Schatzmayr HG, Nogueira RMR, Rosa APAT. An outbreak of dengue vírus at Rio de Janeiro, Memórias do Instituto Oswaldo Cruz 81: 245-246, 1986.

18. Secretaria de Vigilância em Saúde. Dengue. Boletim da semana 04/2005. Ministério da Saúde http://dtr2001.saude.gov.br/svs/epi/dengue/boletim/pdfs/ be_dengue_04_2005.pdf acessado em 15.04.2006, 2005.

19. Secretaria de Vigilância em Saúde. Dengue (cartaz). Ministério da Saúde http://dtr2001.saude.gov.br/svs/epi/dengue/dengue_cartaz.htm, acessado em 15.04.2006, 2006.

20. Streatfield R, Bielby G, Sinclair D. A primary dengue 2 epidemic with spontaneous hemorrhagic manifestations. The Lancet 342:560-561, 1993.

21. Tauil PL. Urbanização e ecologia do Dengue no Brasil. Cadernos de Saúde Pública 17: 99-102, 2001.

22. Teixeira MG, Barreto ML, Costa MCN, Ferreira LDA, Vasconcelos PFC, Cairncross S. Dynamics of dengue virus circulation: a silent epidemic in a complex urban area. Tropical Medicine and International Health 7: 757-762, 2002

23. World Health Organization. A system of world-wide surveillance for vectors Weekly Epidemiological Record 25:73-80, 1972. 